\title{
LETTER TO THE EDITOR Cathepsin B inhibitors block multiple radiation-induced side effects in C. elegans
}

\author{
Cell Research (2019) 29:1042-1045; https://doi.org/10.1038/s41422-019-0247-3
}

\section{Dear Editor,}

Radiotherapy (RT) is one of the major treatments for cancer patients, but its application in cancer therapy has been limited due to deleterious side effects caused by radiation, ${ }^{1}$ which include dry mouth, fatigue, nausea, skin irritation, low blood cell counts, and infertility. These side effects, also called radiation-induced bystander effects (RIBE), are caused by factors released from irradiated cells or tissues, which can act either short- or long-range to impact nearby or distant unexposed cells and tissues to induce genomic instability, stress response, and altered cell proliferation or death. ${ }^{2}$ RIBE thus is a critical factor in determining the efficacy and success of RT in cancer treatment and a major safety concern to people with occupational exposure to radiation, such as aircrew members, medical workers, and laboratory researchers. ${ }^{1,2}$ There is no effective way to prevent adverse side effects caused by radiation and RT. This has been a longstanding medical issue that demands an urgent solution. ${ }^{1}$

Recently, we identified the first RIBE factor in nematodes, CPR-4, which is a homologue of the human cysteine protease cathepsin $B$ (CTSB). ${ }^{3}$ Upon ultraviolet (UV) or ionizing gamma ray (IR) irradiation, CPR-4 is secreted from irradiated animals and becomes the major factor in the medium that can impinge on unirradiated animals to elicit multiple side effects, which include increased embryonic lethality, inhibition of germ cell death, and increased germ cell proliferation. ${ }^{3}$ Moreover, in a C. elegans RIBE model that mimics RT, localized UV irradiation (LUI) at the head of the animal evokes similar CPR-4-dependent side effects at distant unirradiated sites of the same animal (Fig. 1a), leading to increased lethality of unexposed embryos and elevated stress response in the posterior unirradiated region, ${ }^{3}$ which can be detected by the $\mathrm{P}_{\text {hsp-4 }} \mathrm{GFP}$ stress response reporter. Therefore, CPR-4 is an important mediator of both intra- and inter-animal RIBE. ${ }^{3}$ How localized irradiation triggers multiple different, and often detrimental, side effects in distant unexposed cells or tissues is not clear and is a major issue in radiation biology and safety.

To address these important questions, we investigated the possibility that localized irradiation causes side effects in unexposed cells through inducing chromosome instability, especially in cells actively undergoing mitosis, such as $C$. elegans germ cells. Radiation-induced genome instability in unirradiated bystander cells has been documented in culture cells and tissue models, ${ }^{2,4,5}$ but has not been examined rigorously in live animals. The underlying mechanism is unknown, although RIBE-related clastogenic factors, which can induce breakages of chromosomes in unirradiated cells, have been proposed. ${ }^{2}$ To detect DNA damage in $C$. elegans mitotic germ cells, we examined the localization pattern of the DNA damage checkpoint protein, HUS1, a component of the conserved heterotrimeric Rad9, Hus1, and Rad1 complex (also named the 9-1-1 complex), which is loaded onto sites of DNA damage to coordinate checkpoint activation and DNA repair. ${ }^{6,7}$ We inserted the coding sequence of the NeonGreen fluorescent protein into the hus-1 locus to create a hus-1::neongreen fusion knock-in (KI) using the CRISPR/Cas9 gene editing method ${ }^{8}$ and examined if HUS-1::NeonGreen concentrated at sites of DNA damage following whole-body or localized UV irradiation. As expected, whole-body UV irradiation of hus-1:: neongreen $\mathrm{KI}$ animals $\left(100 \mathrm{~J} / \mathrm{m}^{2}\right)$ induced the formation of bright HUS-1::NeonGreen foci in nuclei of multiple mitotic germ cells, which coalesced on chromosomal DNA stained by Hoechst 33342 (Fig. 1b, upper panel), indicating that direct UV irradiation causes many DNA breaks in these germ cell nuclei. Interestingly, localized UV irradiation (LUI) at the head of hus-1::neongreen $\mathrm{KI}$ animals also induced the formation of distinct, bright HUS-1::NeonGreen puncta in nuclei of unexposed mitotic germ cells (Fig. 1a, b, lower panel), which share the cytoplasm in the gonad syncytium. This result indicates that localized irradiation somehow triggers DNA damage in distant unexposed germ cells, probably through RIBE factors. Compared with whole-body UV irradiation, fewer mitotic germ cells in LUI animals had HUS-1::NeonGreen foci (Fig. 1d, e) and markedly less HUS-1::NeonGreen foci were seen in affected mitotic germ cells (Fig. 1b), indicating that the damage to the nuclear DNA of unexposed germ cells induced by RIBE is less severe than that caused by direct UV irradiation. Importantly, LUI-induced HUS-1::NeonGreen foci, but not those caused by whole-body UV irradiation, were dependent on a functional cpr-4 gene, as inactivation of cpr-4 by a deletion mutation, $\operatorname{tm} 3718^{3}$ abolished LUI-induced HUS-1::NeonGreen foci (Fig. 1c-e), but did not prevent DNA damage caused by whole-body UV irradiation that directly caused DNA double-strand breaks in mitotic germ cells. We confirmed this finding using another DNA damage marker, the HSR-9 protein, which is a C. elegans homologue of the human p53 binding protein 1 (53BP1) that plays a key role in DNA damage response and repair in mammalian cells and localizes to DNA double-strand breaks. ${ }^{9}$ HSR-9::GFP fusion expressed from the endogenous $h s r-9$ locus with a gfp knock-in (hsr-9::gfp) generated by the CRISPR/Cas9 method displayed nuclear staining in mitotic germ cells, a large portion of which overlapped with nuclear DNA staining by Hoechst 33342 (Supplementary information, Fig. S1a). ${ }^{9}$ Following whole-body or localized UV irradiation, HSR-9::GFP formed intense foci or patches on chromosomal DNA (Supplementary information, Fig. S1a), with whole-body irradiation producing more bright HSR-9::GFP foci in mitotic germ cells than LUI. Again, the formation of HSR-9::GFP foci after LUI treatment, but not that caused by whole-body irradiation, was dependent on the cpr-4 gene (Supplementary information, Fig. S1b-d). Given the observations that CPR-4 is required for LUI-induced DNA damage in unexposed germ cells and the nature of CPR-4 as a secreted RIBE factor, we tested if CPR-4 by itself could induce DNA damage by incubating hus-1::neongreen $\mathrm{KI}$ and $h s r-9:: g f p$ KI animals with the purified CPR-4 or CTSB protease. In both cases, recombinant CPR-4 or CTSB proteins were sufficient to induce the formation of HUS-1::NeonGreen and HSR-9::GFP foci in mitotic germ cells (Fig. 1f, g). These results indicate that CPR-4 is necessary and sufficient to induce DNA damage in unexposed bystander cells 
a

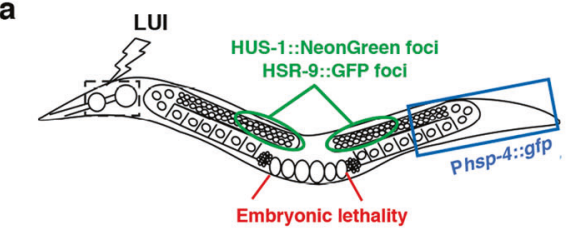

d
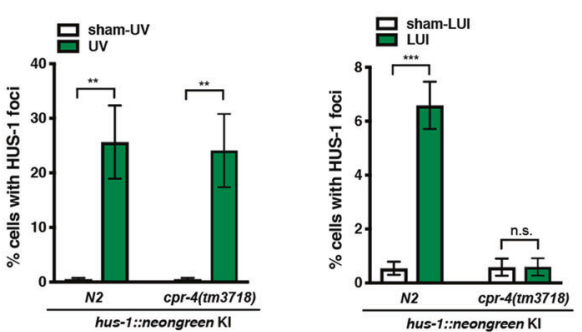

b

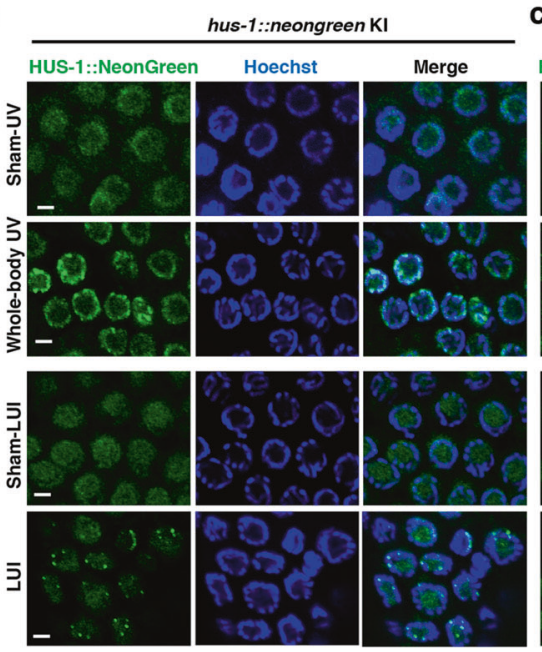

h

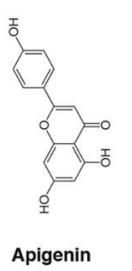

f
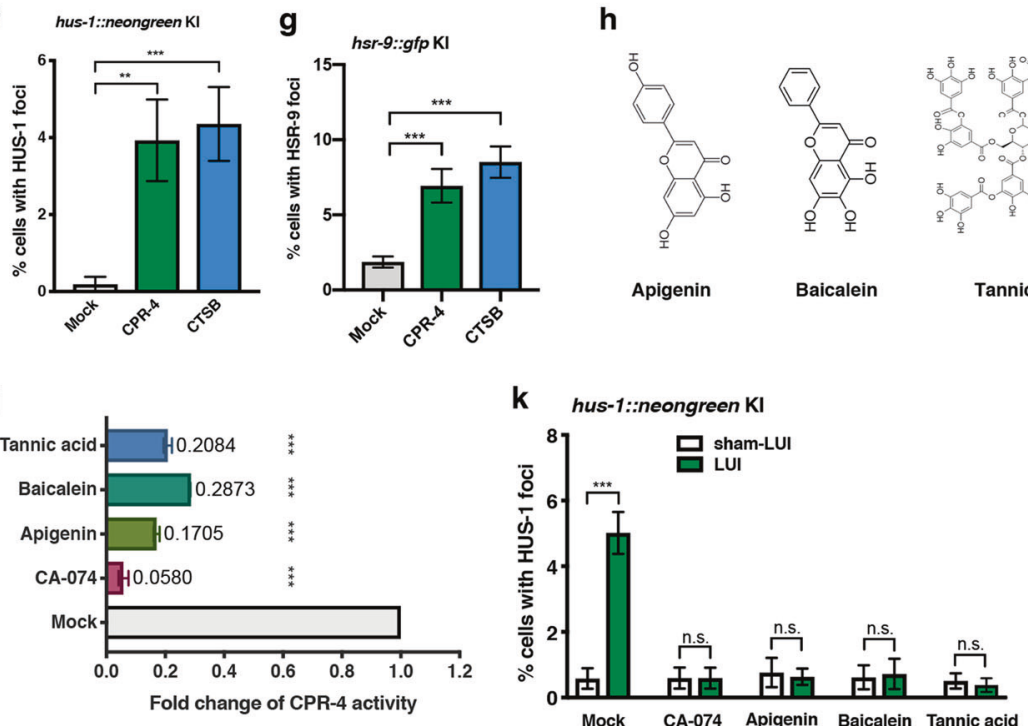

hus-1::neongreen $\mathrm{KI}$; cpr-4(tm3718)
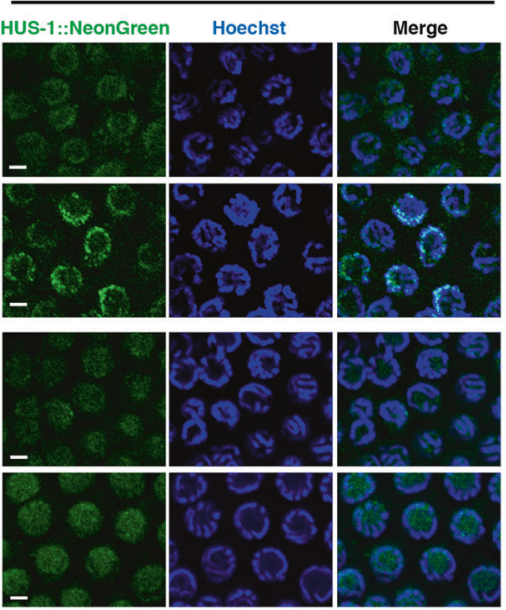

i

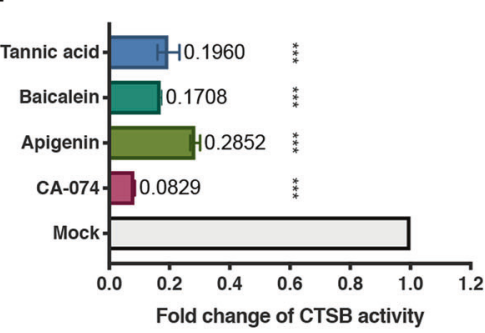

I

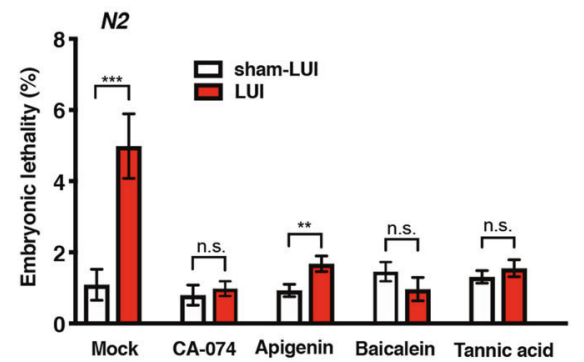

m

Mock
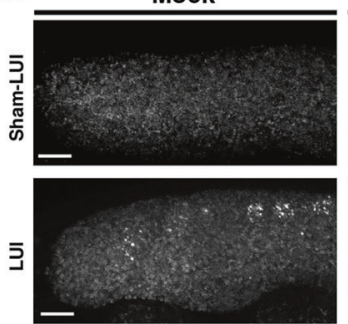

CA-074
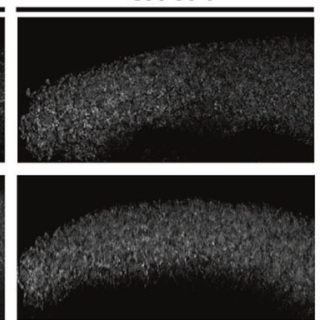

Apigenin
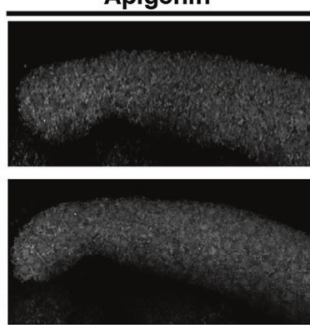

Baicalein

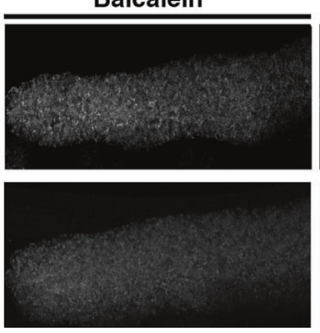

Tannic acid

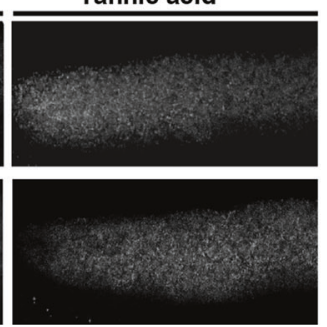

following radiation and is likely the elusive clastogenic factor that evokes DNA damage during RIBE. Taken together, these are the first experimental demonstrations that localized irradiation like radiotherapy can elicit DNA damage in unexposed bystander cells, with the RIBE factor CPR-4 acting as a clastogenic factor.

Given the crucial role of CPR-4 in RIBE and the adverse side effects caused by radiotherapy, which lack a good treatment option, we sought to identify compounds that inhibit the protease activity of CPR-4 and CTSB using a candidate approach, focusing on ten small molecules derived from natural products that have been tested in cancer therapy or show some effects in protecting against radiation (Supplementary information, Fig. S2). ${ }^{10,11}$ Three compounds, apigenin, baicalein and tannic acid, and a known
CTSB inhibitor, CA-074, exhibited good inhibitory activities towards recombinant CTSB and CPR-4 proteases in vitro (Fig. $1 \mathrm{~h} \pm \mathrm{j}$ and Supplementary information, Fig. S3). Although apigenin, baicalein and tannic acid are less potent inhibitors of the CTSB and CPR-4 proteases than CA-074, with the half maximal inhibitory concentrations $\left(\mathrm{IC}_{50}\right)$ ranging from $0.46 \mu \mathrm{M}$ to $96 \mu \mathrm{M}$ compared with $0.013-0.023 \mu \mathrm{M}$ by CA-074 (Supplementary information, Fig. S4), they are the most abundant phenolic compounds found in fruits and vegetables and have shown some anti-cancer activities and low toxicity in animal tests. ${ }^{12-14}$

Using LUI intra-animal RIBE assays, we examined whether apigenin, baicalein, and tannic acid could inhibit different radiation-induced side effects in C. elegans. ${ }^{3}$ These include DNA 
Fig. 1 Novel cathepsin B inhibitors derived from natural products block different radiation-induced side effects in C. elegans. a Schematic presentation of side effect assays in an intra-animal RIBE model. The pharyngeal area of the animal was irradiated by UV and side effects were analyzed in three unexposed tissues as indicated (Methods). These include the formation of HUS-1::NeonGreen or HSR-9::GFP foci in nuclei of mitotic germ cells, increased lethality of embryos, and elevated GFP expression from the $\mathrm{P}_{h s p-4}$ gfp reporter in the posterior region. $\mathbf{b}$, $\mathbf{c}$ DNA damage assays in mitotic germ cells induced by either whole-body UV irradiation or localized UV irradiation (LUI) in the indicated strains. Representative images of HUS-1::NeonGreen, Hoechst 33342 staining of germ cell nuclei, and the merged images are shown. Images were taken $16 \mathrm{~h}$ after whole body UV irradiation or $24 \mathrm{~h}$ after LUI. Scale bars are $2 \mu \mathrm{m}$. d, e The level of DNA damage is quantified as the percentage of germ cells in the mitotic zone that contained bright HUS-1::NeonGreen foci at the time of imaging as described in $\mathbf{b}$, $\mathbf{c}$. In each experiment, at least 15 animals were scored. UV, whole-body UV (d). f, g Recombinant CPR-4 and cathepsin B (CTSB) can induce DNA damage in mitotic germ cells. Laval stage 4 (L4) animals were treated with $2.8 \mu \mathrm{M}$ CPR-4 or CTSB or a buffer control (Mock) for $24 \mathrm{~h}$ before DNA damage was scored as described in $\mathbf{d}$ and Fig. S1c. $\mathbf{h}$ The chemical structures of apigenin, baicalein and tannic acid. $\mathbf{i}, \mathbf{j}$. Protease assays of CTSB and CPR-4 in the presence of $250 \mu \mathrm{M}$ of the indicated compounds (Methods). DMSO (Mock) was used as a negative control. Results are from at least three independent experiments. k, I DNA damage assays (k) and embryo lethality assays (I) following drug treatment and LUI. L1 larvae of the indicated strain were treated with $250 \mu \mathrm{M}$ of apigenin, baicalein, or tannic acid, or $10 \mu \mathrm{M}$ of CA-074 and then subjected to LUI when they reached the L4 stage. Animals were scored $24 \mathrm{~h}$ after LUI. DNA damage was scored as described in e. Embryonic lethality was scored as described previously. ${ }^{3}$ At least 15 adult animals (k) and 900 embryos (I) were scored in each experiment. Data shown are mean \pm s.e.m. n.s., not significant, ${ }^{* *} P<0.01,{ }^{* * *} P<0.001$, two-sided, unpaired $t$-test $(\mathbf{d}-\mathbf{g}, \mathbf{i}-\mathbf{l})$. In $\mathbf{i}$, $\mathbf{j}$, data were compared with Mock. $\mathbf{m}$ Representative images of the mitotic zone of gonads from hus-1::neongreen KI animals pretreated with the indicated compounds and with or without LUI treatment as described in $\mathbf{k}$. Scale bars, $10 \mu \mathrm{m}$

damage in unexposed mitotic germ cells, increased lethality of unexposed embryos, and elevated stress response in unirradiated posterior regions, which can be quantified by monitoring GFP expression from the stress reporter, $\mathrm{P}_{h s p-4} \mathrm{GFP}$ (Fig. 1a). ${ }^{3}$ Treatment of hus-1::neongreen $\mathrm{KI}$ and hsr-9::gfp $\mathrm{KI}$ animals with apigenin, baicalein, tannic acid, or CA-074 all blocked the formation of HUS-1:: NeonGreen or HSR-9::GFP foci in unexposed mitotic germ cells following LUI (Fig. 1k, m and Supplementary information, Fig. S1e, f), similar to that seen in the cpr-4(tm3718) mutant (Fig. 1c, e and Supplementary information, Fig. S1b, d). These results indicate that these compounds inhibit LUI-induced chromosomal DNA damage, a critical RIBE that could lead to other side effects, including genome instability, compromised survival of affected embryos, and stress response. ${ }^{15}$ Indeed, apigenin, baicalein, tannic acid, and CA-074 similarly blocked increased lethality of unexposed embryos (Fig. 1I) and stress response in the posterior regions after LUI treatment (Supplementary information, Fig. S5). Therefore, as novel CTSB and CPR-4 protease inhibitors, apigenin, baicalein, and tannic acid can suppress several different side effects in a $C$. elegans radiation model mimicking radiotherapy in humans. Given the findings that human CTSB and C. elegans CPR-4 exhibit similar protease activities ${ }^{3}$ and that human CTSB mimics CPR-4 to cause DNA damage (Fig. If, g), increased embryonic lethality, and reduced germ cell death in $C$. elegans, ${ }^{3}$ these compounds may similarly inhibit radiotherapy side effects in humans.

Interestingly, apigenin and tannic acid were tested as adjuvant chemotherapeutic agents to alleviate side effects or enhance the potency of chemotherapy. ${ }^{12,13}$ Baicalein was used to mitigate impairment of hippocampal neurogenesis and neurocognitive deficits caused by whole-brain irradiation. ${ }^{14}$ However, their mechanisms of action as adjuvant agents in these therapies are unknown. Our findings provide a plausible mechanism by which these compounds act to reduce side effects of radio- and chemotherapy. Importantly, our study raises alerts to the potentially damaging effect of radiation to the nuclear DNA of distant unexposed cells, which could lead to genetic mutations and other side effects, and suggests possible treatment options to prevent such side effects. Finally, this proof-of-concept drug screen in $C$. elegans demonstrates that the combination of in vitro CTSB inhibitor screens and in vivo verification by $C$. elegans RIBE models is a powerful approach to identify drug leads for alleviating and treating side effects caused by radiotherapy.

\section{ACKNOWLEDGEMENTS}

This work was partly supported by National Institutes of Health grant R35 GM118188 (to D.X.), by Ministry of Science and Technology of China grant
2017YFA0102900, National Natural Science Foundation of China Grant 81741163 , Fujian Province Science and Technology Major Projects grant 2014NZ2002-1, and the 13th Five-Year Plan on Fuzhou Marine Economic Innovation and Development Demonstration City Project (to B.L.), and by fellowships from Fujian Agriculture and Forestry University (to L.Z. and X.W.).

\section{AUTHOR CONTRIBUTIONS}

L.Z. performed the in vitro assays, did the RIBE assays with X.W., collected and analyzed related data. S.L. generated the hus-1::neongreen and hsr-9::gfp KI strains. D.X. conceived and supervised the project, analyzed data, and wrote the manuscript together with L.Z. and B.L.

\section{ADDITIONAL INFORMATION}

Supplementary information accompanies this paper at https://doi.org/10.1038/ s41422-019-0247-3.

Competing interests: The authors declare no competing interests.

$$
\begin{array}{r}
\text { Lingjun Zheng }{ }^{1,2} \text {, Xiaoqi Wu }{ }^{1,2} \text {, Shanshan } \mathrm{Li}^{3} \text {, Bin } \mathrm{Liu}^{1} \text { and } \\
\text { Ding Xue }
\end{array}
$$

${ }^{1}$ College of Food Science, Fujian Agriculture and Forestry University, Fuzhou 350002 Fujian, China; ${ }^{2}$ Department of Molecular, Cellular, and Developmental Biology, University of Colorado, Boulder, CO 80309, USA and ${ }^{3}$ School of Life Sciences, Tsinghua University, Beijing 100084, China

Correspondence: Bin Liu (liubin618@hotmail.com) or Ding Xue (Ding. Xue@Colorado.edu)

\section{REFERENCES}

1. Bentzen, S. M. Preventing or reducing late side effects of radiation therapy: radiobiology meets molecular pathology. Nat. Rev. Cancer 6, 702-713 (2006).

2. Mothersill, C. \& Seymour, C. Radiation-induced bystander effects: past history and future directions. Radiat. Res. 155, 759-767 (2001).

3. Peng, Y. et al. Cysteine protease cathepsin B mediates radiation-induced bystander effects. Nature 547, 458-462 (2017).

4. Lorimore, S. A. et al. Chromosomal instability in the descendants of unirradiated surviving cells after alpha-particle irradiation. Proc. Natl Acad. Sci. USA 95, 5730-5733 (1998).

5. Sedelnikova, O. A. et al. DNA double-strand breaks form in bystander cells after microbeam irradiation of three-dimensional human tissue models. Cancer Res. 67, 4295-4302 (2007).

6. Hofmann, E. R. et al. Caenorhabditis elegans HUS-1 is a DNA damage checkpoint protein required for genome stability and EGL-1-mediated apoptosis. Curr. Biol. 12, 1908-1918 (2002).

7. Parrilla-Castellar, E. R., Arlander, S. J. H. \& Kamitz, L. Dial 9-1-1 for DNA damage: the Rad9-Hus1-Rad1 (9-1-1) clamp complex. DNA Repair 3, 1009-1014 (2004). 
8. Zhao, P., Zhang, Z., Ke, H., Yue, Y. \& Xue, D. Oligonucleotide-based targeted gene editing in C. elegans via the CRISPR/Cas9 system. Cell Res. 24, 247-250 (2014).

9. Wang, B., Matsuoka, S., Carpenter, P. B. \& Elledge, S. J. 53BP1, a mediator of the DNA damage checkpoint. Science 298, 1435-1438 (2002).

10. George, V. C., Dellaire, G. \& Rupasinghe, H. P. V. Plant flavonoids in cancer chemoprevention: role in genome stability. J. Nutr. Biochem. 45, 1-14 (2017).

11. Hazra, B., Ghosh, S., Kumar, A., \& Pandey, B. N., The prospective role of plant products in radiotherapy of cancer: a current overview. Front. Pharmacol. 2, 94, https://doi.org/10.3389/fphar.2011.00094 (2012).

12. Prats, P. M., Garcia, F. G., Diaz, F. M., Mendes, R. A. \& Lopez-Jornet, P. The therapeutic effects of apigenin and dexamethasone on 5 -fluorouracil-induced oral mucositis—a pilot study using a Syrian hamster model. J. Oral. Pathol. Med. 46, 142-147 (2017).

13. Naus, P. J. et al. Tannic acid synergizes the cytotoxicity of chemotherapeutic drugs in human cholangiocarcinoma by modulating drug efflux pathways. J. Hepatol. 46, 222-229 (2007).

14. Oh, S. B. et al. Baicalein attenuates impaired hippocampal neurogenesis and the neurocognitive deficits induced by gamma-ray radiation. Br. J. Pharmacol. 168, 421-431 (2013).

15. Morgan, W. F. Non-targeted and delayed effects of exposure to ionizing radiation: II. Radiation-induced genomic instability and bystander effects in vivo, clastogenic factors and transgenerational effects. Radiat. Res. 159, 581-596 (2003). 